\title{
INFLUENCIA DEL RIESGO PERCIBIDO Y LA NORMA SUBJETIVA EN LA RESERVA DE ALOJAMIENTO POR INTERNET EN UNA MYPE.
}

\author{
${ }^{1}$ Julio Enrique Quispe Tuesta. Mdol , ${ }^{1}$ Ignacio Ruben Tacza Valverde \\ ${ }^{1}$ Universidad Nacional Tecnológica de Lima Sur, Lima, Perú
}

Recibido: 05/09/2021 Revisado: 03/10/2021 Aceptado: 16/11/2021 Publicado: 30/01/2022

\section{Resumen:}

La finalidad de la presente investigacion es determinar la influencia de las características de las personas al realizar de reserva de alojamiento, que puede ser por internet o de manera tradicional, en un establecimiento de hospedaje mype en el norte del Perú. Tomando como referencia la teoria de la acción razonada, se seleccionaron las variables riesgo percibido y norma subjetiva. El procedimiento estadístico utilizado para el análisis de la asociación fue chi cuadrado de independencia con nivel de significancia del 5\% y para determinar la evidencia de la influencia fue la razón de ventaja o estimación de riesgo, mediante la prueba de Odds Ratio con intervalo de confianza del 95\%. Con estas dos pruebas se encontro que existe asociación entre el riesgo percibido bajo y la norma subjetiva baja con la reserva de alojamiento por internet, teniendo una mayor influencia el riesgo percibido y una influencia moderada la norma subjetiva.

Palabras clave: riesgo percibido, norma subjetiva, reserva de alojamiento por internet.

\begin{abstract}
This study aims to determine personal influences of people for making an accommodation reservation by internet or traditional presential channel, in a northern peruvian hostel. The variables perceived risk and subjective norm were selected from the theory of reasoned action. The statistical procedure used for the association analysis was independence chi-square with 5\% significance level and the advantage ratio or risk estimate to determine the influence evidence, using the odds ratio test with $95 \%$ confidence interval. The results shown there is an association between the low perceived risk and the low
\end{abstract}


subjective norm with the reservation of accommodation online, having a greater influence on the perceived risk and a moderate influence on the subjective norm.

Key Words: perceived risk, subjective norm, internet reservation

\section{INTRODUCCIÓN}

Investigaciones relacionadas al comercio electrónico en el sector turismo en el Perú son escasos, los que existen proporcionan datos estadísticos muy genéricos, relacionados principalmente a las grandes empresas.

Las mypes en el Perú representan el 99\% de las empresas nacionales y genera más del $70 \%$ de los empleos, a pesar de ello, su desarrollo no siempre va de la mano con el mundo globalizado en que vivimos, mostrando diferencias para sus sectores, así pues, el comercio electrónico del sector turismo no está al nivel de desarrollo como del sector de comidas, por citar un ejemplo. Por ello, la presente investigación trata de encontrar razones porque la reserva de alojamiento por internet no está desarrollando en el nivel esperado, y específicamente identificar los factores que condicionan la decisión de reserva de alojamiento por internet en los usuarios de las mypes de Perú.

Se encontraron investigaciones que tratan de explicar el comportamiento de la persona al momento de realizar transacciones electrónicas, la mayoría de ellos aplicaron el modelo propuesto por Icek Ajzen, llamado Teoría de la Acción Razonada, la cual, pretende predecir el comportamiento de una persona ante situaciones determinadas, a través de variables como el riesgo que percibe el individuo y la norma subjetiva del círculo de personas o medios que considera importante para su decisión.

La variable Riesgo Percibido es una adaptación de la variable control percibido del modelo teórico de Icek Ajzen (1991), y se define como una potencial incertidumbre de posibles resultados negativos (Nunkoo \& Ramkissoon, 2013). Investigaciones previas constatan la influencia del control percibido en el comportamiento de uso de servicios electrónicos, como los entornos virtuales de aprendizaje, servicios bancarios online, los juegos online o los servicios del móvil. Centrándonos en el ámbito turístico, el estudio de

Ruiz y Sanz (2010) evidencia que el control percibido, definido como el resultado de las 
creencias del individuo sobre la presencia o ausencia de las habilidades, oportunidades y recursos necesarios para la compra de servicios.

La norma subjetiva recoge la influencia de las opiniones de otras personas como familia, amigos, compañeros de trabajo, etc., en el comportamiento del individuo. La norma subjetiva de una persona con relación a su conducta y/o comportamiento está basada en sus creencias predominantes, y pueden ser de dos tipos, las creencias normativas que son el conjunto de creencias que influyen en un individuo y proviene de un grupo específico de personas y la normativa social o también llamada normativa subjetiva que se adquiere del entorno. La norma subjetiva puede ejercer presión en una persona, para determinar su conducta ante un caso específico, independientemente de la actitud y creencias que pueda tener (Ajzen \& Fishbein, 1975).

Inés Álvarez (2016), en la revista Forbes, cuenta que el fenómeno Airbnb se inicia en el año del 2008, la plataforma Airbnb permite alquilar inmuebles particulares tales como: casas, departamentos o habitaciones, a precios mucho más económicos, brindando a los propietarios la oportunidad de ganar dinero extra.

Booking.nl como su nombre original comenzó a operar en 1996, con una sola oficina y un solo empleado, incluido su creador Geert-Jan Bruisnma. En el Perú, Booking.com lidera la preferencia de los peruanos frente a su competidor directo, Airbnb. En el departamento de Lambayeque, que es donde se realiza la investigación, también se evidencia la preferencia (Google Trends, 2019).

El sitio web Google (2018), señala que el $94 \%$ de peruanos que decide realizar una compra por internet tomando como referencia lo que investiga por internet, un $78 \%$ antes de decidir ya vio un video del producto que pensaba comprar.

Sanz, Ruiz, Pérez y Hernández (2014), en su investigación Influencia de la cultura en el comportamiento de compra online de productos turísticos, trata de medir la influencia de la norma subjetiva, la actitud y el control percibido en la intención de compra en usuarios internautas de México y España. Los resultados encontrados muestran que la Norma Subjetiva tiene una influencia de $\beta(\mathrm{t})=0.402$, el Control Percibido tiene una influencia de $\beta(\mathrm{t})=0.276$, y la Actitud tiene una influencia de $\beta(\mathrm{t})=0.052$, los tres sobre la intención de compra, Por lo tanto, la Norma Subjetiva y el Control Percibido tiene una influencia sobre la intención de compra de los internautas. 
Para presente investigación, se tomó como muestra una mype de alojamiento ubicado en la ciudad de Chiclayo, Hostal Satélite, una microempresa que tiene 4 trabajadores, de categoría dos estrellas, con alrededor de 20 años de servicio, que ha tenido varias administraciones. En el año 2016, decidieron ingresar al mundo del comercio electrónico a través de internet, y escogieron la plataforma digital Booking.com (Booking.com, 2016), buscador líder mundial para reservas de alojamiento; para anunciar y publicitar la reserva de su alojamiento, con el fin de poder ampliar la demanda de sus instalaciones a través de ese nuevo canal, Los resultados fueron favorables, tanto así, que Hostal Satélite, fue posicionado en primer lugar por Booking.com en Chiclayo; reconocimiento que se otorga al establecimiento con mejores calificaciones de los huéspedes por su calidad de servicio registrado por sus huéspedes, superando incluso a hoteles cinco estrellas y cadenas internacionales.

El objetivo de la investigación es evidenciar la influencia del riesgo percibido y la norma subjetiva en la reserva de alojamiento por internet en una mype de Perú, en el año 2019.

La presente investigación servirá a las personas dedicadas a ofrecer servicios de consultoría y/o asesoría en oferta de alojamiento pyme, y a los propietarios de las mypes de alojamiento, brindándoles una información de la realidad de una mype de provincia y que les permitirá tomar acciones para incrementar el uso de internet para las reservas de alojamiento, que apoyen a asegurar una mayor ocupación de sus habitaciones y mejorar sus servicios con una proyección al cliente.

Las hipótesis son El riesgo percibido y la norma subjetiva influyen en la reserva de alojamiento por internet en una mype de Perú. Existe asociación entre el riesgo percibido bajo y la reserva de alojamiento por internet en una mype de Perú. Existe asociación entre la norma subjetiva baja y la reserva de alojamiento por internet en una mype de Perú.

\section{METODOS}

La población para el estudio son los usuarios de las mype de alojamiento del norte del Perú, conformadas por hostales de dos y tres estrellas. Y la muestra está conformada por los usuarios del Hostal Satélite de Chiclayo que reservaron y se alojaron durante el tercer trimestre, los meses de julio, agosto y setiembre de 2019 y aceptaron llenar la encuesta en la recepción del hostal. 
El tipo de muestra que se utilizo es el muestreo aleatorio sistemático, según la definición de José Supo, en este caso no se cuenta con marco muestral, no existe un N que corresponde a la población, y por ello se toma como muestra a los usuarios que llegan dentro de un periodo seleccionado, usuarios que llegan aleatoriamente (Supo \& Zacarías, 2020).

Los datos de los usuarios levantados mediante las encuestas nos permiten, mediante métodos de reducción del software estadístico a usar, obtener tres variables categóricas dicotómicas, que se muestran en la Tabla 1.

Tabla 1.

Variables dicotómicas y sus categorías

\begin{tabular}{lll}
\hline \multicolumn{1}{c}{ VARIABLE } & \multicolumn{1}{c}{ CATEGORIA 1 } & \multicolumn{1}{c}{ CATEGORIA 2 } \\
\hline Reserva de & Reserva de alojamiento & Reserva de alojamiento \\
alojamiento & por Internet & Tradicional \\
Riesgo Percibido & Riesgo percibido Bajo & Riesgo percibido Alto \\
Norma Subjetiva & Norma Subjetiva Baja & Norma Subjetiva Alta \\
\hline
\end{tabular}

Tabla 2

Herramientas usadas en el análisis estadístico de la investigación

\begin{tabular}{lll}
\hline \multicolumn{1}{c}{ OBJETIVO } & HERRAMIENTA & \multicolumn{1}{c}{ INDICADOR } \\
\hline Describir Variables & Frecuencias & Porcentaje \\
Asociar de Categorías & Chi Cuadrado de & Chi Cuadrado \\
de variables & Independencia & P-valor \\
Medir la Fuerza de & Odds Ratio & Razón de ventajas \\
Asociación & & \\
\hline
\end{tabular}

El análisis de los datos obtenidos mediante las encuestas antes indicadas, se procesaron haciendo uso del software SPSS, versión 22. Y en la Tabla 2 se muestran las herramientas estadísticas utilizadas en el software y los indicadores en cuyos valores nos apoyamos para tomar la decisión de aceptar las hipótesis. 


\section{RESULTADOS}

La muestra para el presente estudio estuvo compuesta por 133 usuarios encuestados en el tercer trimestre del año 2019, julio, agosto y setiembre, en la Figura 1 se muestran las frecuencias de las categorías de reserva de alojamiento.

\section{Figura 1}

Porcentaje por categoría de Reserva de alojamiento

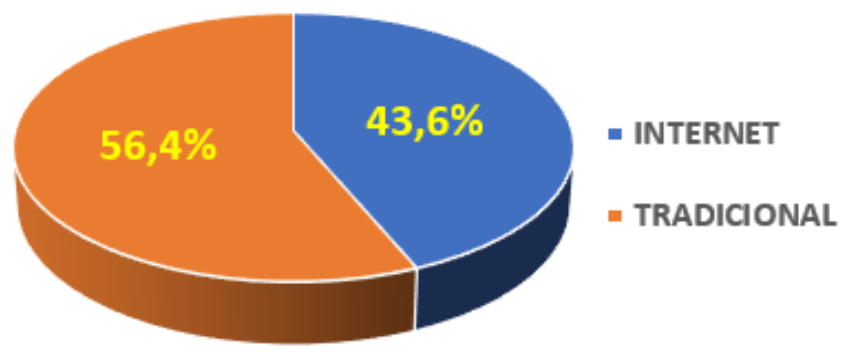

En la Figura 2 se muestran los porcentajes de las categorías del riesgo percibido de las personas encuestadas.

\section{Figura 2}

Porcentaje por categoría de Riesgo percibido

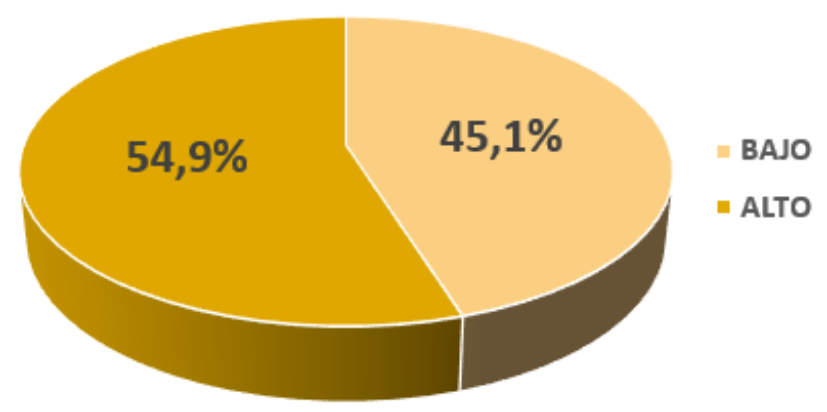

Y en la figura 3 se observa el porcentaje de personas con norma subjetiva baja y alta.

\section{Figura 3}

Porcentaje por categoría de Norma Subjetiva 


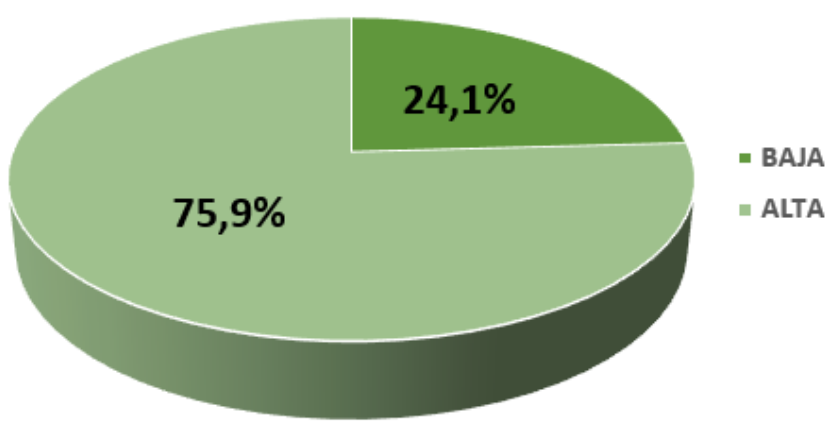

\section{Riesgo Percibido Bajo y Reserva de alojamiento por Internet}

Para determinar la asociación del riesgo percibido bajo con la reserva de alojamiento por internet en una mype de Perú, se procesan los datos para responder la interrogante ¿Existe asociación entre el riesgo percibido bajo y la reserva de alojamiento por internet?, En la Tabla 3, se muestran la hipótesis nula H0 y la hipótesis de la investigación H1, el nivel de significancia, el valor de Chi Cuadrado y el P-valor que el software nos da como resultado al usar la herramienta Prueba de Chi cuadrado de independencia.

\section{Tabla 3}

Asociación de Riesgo percibido Bajo con Reserva de alojamiento por Internet

\begin{tabular}{ll}
\hline \multicolumn{1}{c}{ RUBRO } & \multicolumn{1}{c}{ DESCRIPCIÓN - VALORES } \\
\hline Hipótesis H0 & $\begin{array}{l}\text { No existe asociación entre el riesgo percibido } \\
\text { bajo y la reserva de alojamiento por internet }\end{array}$ \\
Hipótesis H1 & $\begin{array}{l}\text { Existe asociación entre el riesgo percibido } \\
\text { bajo y la reserva de alojamiento por internet }\end{array}$ \\
Alfa - Nivel de & $\alpha=5 \%=0.05$ \\
significancia & \\
Prueba de Chi & Chi Cuadrado $=39.275$ \\
cuadrado de & P-Valor $=3.681$ E-10 $=0.000 \%$ \\
independencia & \\
\hline
\end{tabular}


De la Tabla 3 se puede concluir que: con una probabilidad de error del $0.000 \%$ existe asociación entre el riesgo percibido bajo y la reserva de alojamiento por internet, por lo tanto, se acepta la hipótesis de la investigación H1: Existe asociación entre el riesgo percibido bajo y la reserva de alojamiento por internet.

También de los resultados que nos proporciona el SPSS, cuando se analiza tablas cruzadas para la aplicación del Chi cuadrado, se tiene los porcentajes que se relacionan entre las categorías de las variables en estudio, se muestran en la Figura 4.

\section{Figura 4. Porcentaje de las categorías de Riesgo percibido y reserva de alojamiento}

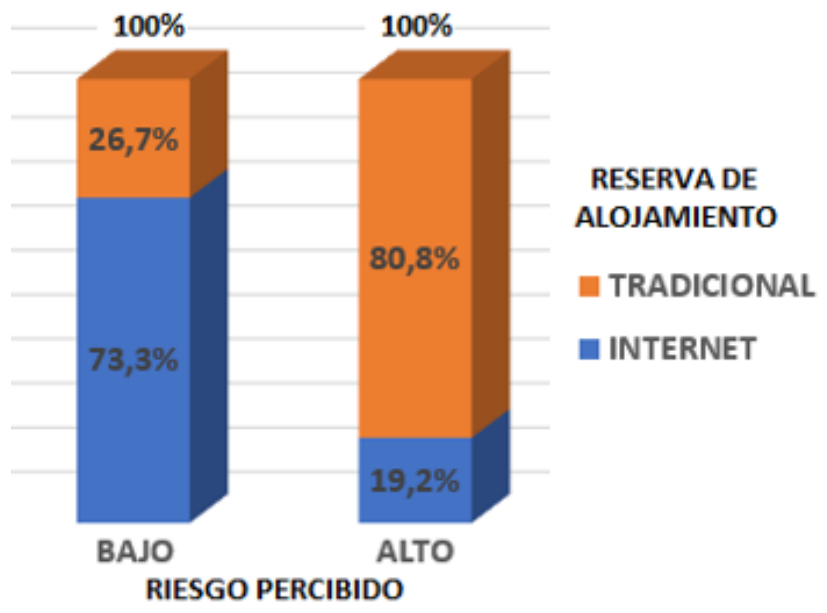

\section{Norma Subjetiva Baja y Reserva de alojamiento por Internet}

Para determinar la asociación de la norma subjetiva baja con la reserva de alojamiento por internet en una mype de Perú, se procesan los datos para responder la interrogante ¿Existe asociación entre la norma subjetiva baja y la reserva de alojamiento por internet? 


\section{Tabla 4.}

Determinar asociación de Norma Subjetiva Baja con Reserva de alojamiento por internet

\begin{tabular}{ll}
\hline \multicolumn{1}{c}{ RUBRO } & \multicolumn{1}{c}{ DESCRIPCIÓN - VALORES } \\
\hline Hipótesis H0 & $\begin{array}{l}\text { No existe asociación entre la norma subjetiva } \\
\text { baja y la reserva de alojamiento por internet }\end{array}$ \\
Hipótesis H1 & $\begin{array}{l}\text { Existe asociación entre la norma subjetiva } \\
\text { baja y la reserva de alojamiento por internet }\end{array}$ \\
Alfa - Nivel de & $\alpha=5 \%=0.05$ \\
significancia & \\
Prueba de Chi & Chi Cuadrado $=4.259$ \\
cuadrado de & P-Valor $=0.039=3.9 \%$ \\
independencia & \\
\hline
\end{tabular}

En la Tabla 4, se muestran la hipótesis nula H0 y la hipótesis de la investigación H1, el nivel de significancia, el valor de Chi Cuadrado y el P-valor que el software nos da como resultado al usar la herramienta Prueba de Chi cuadrado de independencia También de la Tabla 4 se puede concluir que: con una probabilidad de error del 3.9\% existe asociación entre la norma subjetiva baja y la reserva de alojamiento por internet, por lo tanto, se acepta la hipótesis de la investigación H1: Existe asociación entre la norma subjetiva baja y la reserva de alojamiento por internet.

El SPSS también brinda otros resultados, cuando se analiza tablas cruzadas para la aplicación del Chi cuadrado, y se tiene los porcentajes que se relacionan entre las categorías de las variables en estudio, se muestran en la Figura 5.

Figura 5. Porcentaje de las categorías de Norma Subjetiva y reserva de alojamiento 


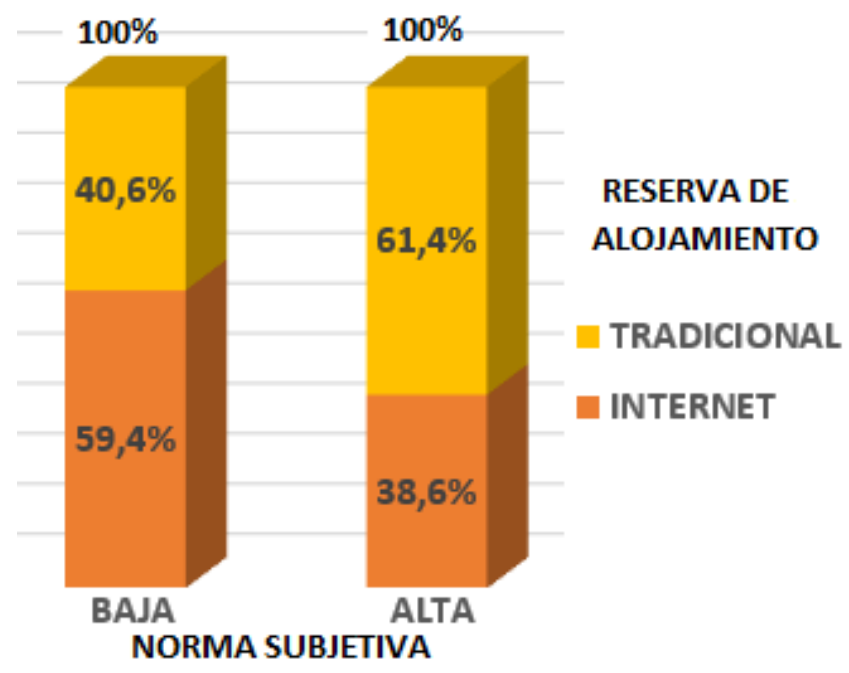

\section{Evidencia de la influencia de las variables en la Reserva de alojamiento por Internet}

Para evidenciar la influencia del riesgo percibido y la norma subjetiva en la reserva de alojamiento por internet, se obtendrá la razón de ventaja o estimación de riesgo para cada una de las categorías asociadas con la categoría reserva de alojamiento por internet.

Después de realizar el análisis estadístico para responder a la interrogante ¿Cuál es la Razón de Ventaja u Odds Ratio entre las categorías asociadas con la categoría reserva de alojamiento por internet? Se obtuvo:

\section{Tabla 5.}

\section{Razón de Ventaja de las categorías de las variables}

\begin{tabular}{cccc}
\hline Variable (Categorías) & \multicolumn{3}{c}{ ODDS Ratio } \\
& Valor & Inferior & Superior \\
\hline Riesgo Percibido (Bajo / Alto) & 11.58 & 5.12 & 26.22 \\
Norma Subjetiva (Baja / Alta) & 2.32 & 1.03 & 5.23 \\
\hline
\end{tabular}

En la Tabla 5 se tiene los resultados de aplicar la herramienta estadística Riesgo correspondiente a Odds Ratio, con intervalo confianza del 95\%, en el SPSS, y en base a ella podemos indicar, que existe una influencia en sentido positivo para la reserva de alojamiento por internet, de acuerdo con lo siguiente:

Las personas que tiene riesgo percibido bajo aumentan la probabilidad de realizar una reserva de alojamiento por internet en 11.6 veces más que las personas que tiene riesgo percibido alto. 
Las personas que tiene norma subjetiva baja aumentan la probabilidad de realizar una reserva de alojamiento por internet en 2.3 veces más que las personas que tiene norma subjetiva alta.

\section{DISCUSION}

Las personas con Riesgo percibido bajo tiene una probabilidad muy grande de hacer una reserva de alojamiento por internet, que las personas con Riesgo percibido Alto, 11.6 veces más, y esto es lo razonable, pues las personas con bajo riesgo percibido son más arriesgadas y se atreven a probar nuevos métodos en todas las actividades de su vida y por lo tanto prueban el uso de internet para las compras, las reservas de alojamiento, para pagar servicios, etc. Las personas con alto riesgo percibido son temerosas y piensan muchas veces antes de cambiar sus hábitos de vida.

La teoría de la conducta planeada fue propuesta por Icek Ajzen (1991), y se ha utilizado con éxito para predecir y explicar una amplia gama de comportamientos y uno de sus componentes es el riesgo percibido que se refiere a la percepción que tiene una persona de la facilidad o dificultad de realizar el comportamiento de interés, en este caso el uso del internet y el crecimiento de las reservas por internet va a tomar tiempo y que depende de la percepción de las personas, de la facilidad, la dificultad, la transparencia, la seguridad y el nivel de desconfianza, que son aspectos que contribuyen al riesgo percibido de una persona.

Las personas con Norma subjetiva baja tiene una probabilidad mayor, aunque sea solo moderada de hacer una reserva de alojamiento por internet, que las personas con Norma subjetiva Alta, 2.3 veces más, y esto podemos considerar a que son personas poco influenciables, que tienen sus convicciones definidas y lentamente incorporan las nuevas experiencias propias o de terceros a su práctica personal diaria, mientras que las personas con Norma subjetiva alta son influenciables tanto positivamente como negativamente, y por los resultados del presente estudio, estimamos que los comentarios y las recomendaciones de las amistades y familiares son para tener cuidado con las malas prácticas de los delincuentes cibernéticos, principalmente en estafas e incumplimiento de compromisos.

Google (2018), señala que el 94\% de peruanos decide realizar una compra por internet tomando como referencia lo que investiga por internet, un $78 \%$ antes de decidir ya vio un 
video del producto que pensaba comprar, entonces la decisión de compra de un bien o de acceso a un servicio está asociada a la norma subjetiva.

Sanz, Ruiz, Pérez y Hernández (2014), en su investigación "Influencia de la cultura en el comportamiento de compra online de productos turísticos", considera la influencia de la norma subjetiva, la actitud y el control percibido en la intención de compra en usuarios de México y España. Los resultados muestran que la Norma Subjetiva tiene mayor influencia sobre la intención de compra, seguido por el Control Percibido y finalmente la Actitud.

La norma subjetiva baja podemos considerar como la posibilidad de recibir poca influencia externa en sus decisiones, y, además, si esta influencia externa transmite poco o casi nada del mundo digital, las compras por internet serán realizadas por solo el $5 \%$ de la población del Perú como se indicó antes.

\section{CONCLUSIONES}

Las variables de esta investigación son tres y del tipo de variables categóricas dicotómicas: 1. La variable Reserva de Alojamiento tiene dos categorías: Reserva de alojamiento por Internet y Reserva de alojamiento Tradicional. 2. La variable Riesgo Percibido tiene dos categorías: Riesgo Percibido Bajo y Riesgo Percibido Alto. 3. La variable Norma Subjetiva, también tiene dos categorías: Norma Subjetiva Baja y Norma Subjetiva Alta.

2. La muestra que se tuvo para el presente estudio fue de 133 usuarios de la Mype de Alojamiento, entre los usuarios encuestados el 56.4\% realizó su reserva por los medios tradicionales y el $43.6 \%$ realizó la reserva por internet, el $54.9 \%$ tiene riesgo percibido alto y $45.1 \%$ riesgo percibido bajo, y el $75.9 \%$ norma subjetiva alta y el $24.1 \%$ norma subjetiva baja.

3. Del análisis estadístico se determina que existe asociación entre las categorías de las variables en el siguiente modo: 1. Existe asociación entre el Riesgo Percibido Bajo y la Reserva de alojamiento por Internet, con una probabilidad de error del 0.000\%. 2. Existe asociación entre la Norma Subjetiva Baja y la Reserva de alojamiento por Internet, con una probabilidad de error del $3.9 \%$.

4. La influencia del Riesgo Percibido en la Reserva de alojamiento por Internet es muy grande, que se evidencia en el análisis estadístico con un nivel de significancia del 5\%, y con 
un nivel de confianza del 95\% para la razón de ventaja o estimación de riesgo, con el siguiente resultado: Las personas que tiene riesgo percibido bajo aumentan la probabilidad de realizar una reserva de alojamiento por internet en 11.6 veces más que las personas que tiene riesgo percibido alto.

5. La influencia de la Norma Subjetiva en la Reserva de alojamiento por Internet es moderado, que se evidencia en el análisis estadístico con un nivel de significancia del 5\%, y con un nivel de confianza del 95\% para la razón de ventaja o estimación de riesgo, con el siguiente resultado: Las personas que tiene norma subjetiva baja aumentan la probabilidad de realizar una reserva de alojamiento por internet en 2.3 veces más que las personas que tiene norma subjetiva alta. 


\section{BIBLIOGRAFÍA}

Ajzen, I. (1 de Diciembre de 1991). The theory of planned behavior. Organizational Behavior and Human Decision, 50(2), 179-211.

Ajzen, I., \& Fishbein, M. (1975). Belief, Attitude, Intention, and Behavior: An Introduction to Theory and Research. Massachusetts: Addison-Wesley.

Alvarez, I. (31 de Marzo de 2016). FORBES. Recuperado el 17 de Noviembre de 2018, de La increible historia de Airbnb: http://forbes.es/business/8618/la-increible-historiade-airbnb/

Booking.com. (15 de Enero de 2016). Booking.com. Recuperado el 4 de Setiembre de 2019, de Booking.com: https://www.booking.com/hotel/pe/hostalsatelite.es.html?aid=304142;label=gen173nr1FCAEoggI46AdIM1gEaLEBiAEBmAEKuAEYyAEM2AEB6AEBAELiAIBqAIEuAK50cnvBcACAQ;sid=2a4ac8f3aa33a182bf3a7301de4d75ef;all_sr_bl ocks=155837417_129984573_2_1_0; checkin=2019-12-25;che

Google. (21 de Agosto de 2018). El Comercio. Recuperado el 31 de Octubre de 2018, de https://elcomercio.pe/economia/peru/google-94-peruanos-decide-compra-basadoinvestiga-internet-noticia-549163

Google Trends. (12 de Diciembre de 2019). Google Trends. Obtenido de https://trends.google.es/trends/explore?cat=955\&date=2016-01-01\%202019-12$12 \& \mathrm{geo}=\mathrm{PE} \& \mathrm{q}=\% 2 \mathrm{Fm} \% 2 \mathrm{~F} 0 \mathrm{svqyn} 7, \% 2 \mathrm{Fm} \% 2 \mathrm{~F} 0 \mathrm{yxzc} 1 \mathrm{z}$

Nunkoo, R., \& Ramkissoon, H. (2013). Travelers' E-Purchase Intent of Tourism Products and Services. Journal of Hospitality Marketing \& Management, 505-529.

Ruiz, C., \& Sanz, S. (2010). Análisis del comportamiento postcompra de servicios turísticos en Internet. Un estudio basado en la teoría del comportamiento planificado. XXIV Congreso Anual de la Academia Europea de Dirección y Economía de la Empresa., (págs. 9-11). Santiago de Compostela.

Sanz Blas, S., Ruiz Mafé, C., Perez Perez, I., \& Hernandez Ortega, B. (2014). La Influencia de la cultura en el comportamiento de compra online de productos turísticos. El caso de España y México. Innovar, 24(54), 153-165.

Supo, J., \& Zacarías, H. (2020). Metodología de la Investigación Científica (Sexta ed.). 